RAEIC, Revista de la Asociación Española de Investigación

\title{
Participación de la audiencia en la rendición de cuentas de los medios de comunicación: instrumentos de accountability y su percepción por parte de los ciudadanos españoles
}

Audience participation for media accountability: instruments and their perception by Spanish citizens

\author{
Ramon-Vegas, Xavier \\ Universitat Pompeu Fabra (UPF) \\ xavier.ramon@upf.edu \\ Mauri-Ríos, Marcel \\ Universitat Pompeu Fabra (UPF) \\ marcel.mauri@upf.edu
}

Forma de citar este artículo:

Ramon-Vegas, X., y Mauri-Ríos, M. (2020). “Participación de la audiencia en la rendición de cuentas de los medios de comunicación: instrumentos de accountability y su percepción por parte de los ciudadanos españoles", RAEIC, Revista de la Asociación Española de Investigación de la Comunicación, vol. 7, núm. 13, 50-76.

https://doi.org/10.24137/raeic.7.13.3

RAEIC, Revista de la Asociación Española de Investigación de la Comunicación vol. 7, núm. 13 (2020), 50-76 


\section{Resumen:}

En escenario actual la rendición de cuentas de los medios de comunicación cobra una importancia de primera magnitud para ayudar a recuperar el prestigio del periodismo ante la opinión pública. La rendición de cuentas se sustenta en tres pilares fundamentales: la transparencia informativa, la autorregulación y la participación del público. Tanto desde dentro de las empresas periodísticas como de forma externa a ellas se ha desarrollado un amplio abanico de instrumentos tradicionales e innovadores que tienen como finalidad velar por la responsabilidad de los medios y la calidad del producto informativo resultante. La presente investigación sistematiza y clasifica los instrumentos de accountability que fomentan la transparencia, la autorregulación y la participación de los usuarios. Posteriormente, se examinan y se ejemplifican 12 instrumentos que facilitan la participación. Finalmente, a través de 6 grupos de discusión se analiza la percepción de los ciudadanos españoles sobre estos últimos mecanismos.

Palabras clave: rendición de cuentas, medios de comunicación, instrumentos, percepción, ciudadanos.

\section{Abstract:}

In the current landscape media accountability is of outmost importance to help restoring the prestige of journalism in the eyes of the public opinion. Accountability is based on three cornerstones: transparency, self-regulation, and public participation. Media companies and other agents beyond them have developed a wide range of established and innovative instruments whose main objective is to look after the responsibility of media and the quality of news output. This research systematizes and classifies the accountability instruments that promote transparency, self-regulation, and users' participation. Afterwards, 12 instruments that facilitate participation are examined and exemplified. Finally, through 6 focus groups we analyse Spanish citizens' perceptions of the aforementioned mechanisms.

Keywords: accountability, media, instruments, perception, citizens. 


\section{INTRODUCCIÓN}

El periodismo atraviesa un momento convulso, caracterizado por la concentración de medios, la aceleración de los ciclos informativos, la transformación tecnológica y su impacto en los modelos de negocio, la creciente polarización de los contenidos y la expansión de fenómenos como el clickbait o la desinformación (Luengo, Maciá-Barber y Requejo-Alemán, 2017; Waisbord, 2019; Zelizer, 2018). Estos factores, combinados con la agudización de problemas clásicos en la profesión-como las injerencias por parte de los poderes políticos y económicos (Alsius, 2010)- ha provocado una fuerte crisis de confianza y credibilidad en este ámbito profesional (Hanitzsch, Van Dalen y Steindl, 2018). Como señalan García-de-Torres y Farmer (2017, p.155), "la sociedad, crítica, parece poco dispuesta a confiar en los medios periodísticos, lo que hace de esta profesión un objetivo más vulnerable ante los ataques externos". Dada la complejidad del escenario actual, la rendición de cuentas cobra una importancia de primera magnitud para ayudar a recuperar el prestigio del periodismo ante la opinión pública (Bertrand, 2018).

Se entiende por rendición de cuentas (media accountability) "la voluntad de los medios a responder frente a la sociedad por la actividad que llevan a cabo" (Mauri-Ríos y Ramon-Vegas, 2015, p.381). La rendición de cuentas tiene varios propósitos esenciales: (1) "monitorizar, controlar, criticar y analizar la calidad de la información periodística" (Ramon-Vegas, Mauri-Ríos y Alcalá-Anguiano, 2016, p.102); (2) "prevenir o limitar el daño que los medios puedan causar" (McQuail, 1997, p.525); (3) velar por la independencia, la transparencia y pluralismo de los medios de comunicación; y (4) ayudar a "cultivar la educación mediática de la audiencia" (van der Wurff y Schönbach, 2014, p.123).

La complejidad del escenario actual requiere que todos los actores involucrados en el proceso informativo -periodistas, ciudadanos y organizaciones independientesparticipen de forma activa en la tarea de fomentar la rendición de cuentas, que se 
sustenta en tres pilares fundamentales: la transparencia informativa, la autorregulación y la participación del público (Ramon-Vegas, Mauri-Ríos y Alcalá-Anguiano, 2016).

En primer lugar, la transparencia puede entenderse como "la apertura de información por parte de las instituciones públicas y de otras entidades sin restricciones, de forma que los datos que se aporten sean fiables y actualizados para que la ciudadanía pueda tomar decisiones responsables" (Díez-Garrido, Campos-Domínguez y Calvo, 2019, p.85). En el ámbito periodístico, la transparencia se refiere más concretamente a la necesidad que los medios proporcionen "públicamente información corporativa acerca de sí mismos, lo que permite a la audiencia comprender los principios y procesos editoriales, la estructura organizativa y la situación financiera de los medios de comunicación" (Ramon-Vegas, Mauri-Ríos y Alcalá-Anguiano, 2016, p.103).

En segunda instancia, la autorregulación hace referencia a las "normas o pautas de conducta que los medios de comunicación y periodistas se autoimponen como compromiso con el público para realizar una comunicación rigurosa, responsable y ética" (Ramon-Vegas, Mauri-Ríos y Alcalá-Anguiano, 2016, p.103). Esta comunicación responsable incluye proporcionar a los ciudadanos información contextualizada y en profundidad, que les permita participar activamente en la vida democrática con plenas garantías (Christians et al., 2009). La autorregulación, preferible a la regulación legal, es esencial para recuperar la confianza de los ciudadanos en el periodismo (Real Rodríguez, 2018).

El tercer valor clave de la rendición de cuentas es la participación. Desde dentro y fuera de los medios se han puesto en marcha iniciativas "que incentivan el contacto directo con el público para facilitar su participación activa" (Ramon-Vegas, Mauri-Ríos y AlcaláAnguiano, 2016, p. 103) en los procesos de producción, revisión y crítica periodística. Esta voluntad de conexión permanente se potencia mediante múltiples iniciativas de carácter presencial, así como a través de nuevos mecanismos orientados a la 'deliberación digital' (Masip, Ruiz-Caballero y Suau, 2019) que "no sólo refuerzan el vínculo medio-usuario sino que otorgan a los lectores el papel de corresponsables del 
compromiso de llevar a cabo un periodismo riguroso y de calidad" (Mauri-Ríos y RamonVegas, 2015, p.387).

Tanto desde dentro de los medios de comunicación como de forma exógena a los mismos se ha desarrollado un amplio abanico de instrumentos que fomentan estas tres dimensiones clave con el convencimiento que "si el periodismo no se autorregula, perderá su credibilidad y su habilidad para promover la democracia" (Ferruci, 2019, p.290). Bertrand (2018) señala que los instrumentos de rendición de cuentas (media accountability instruments) son aquellos mecanismos sin intervención estatal-es decir, no controlados por los gobiernos e impulsados por las empresas, periodistas, organizaciones y ciudadanos- que tienen como finalidad velar por la responsabilidad de los medios y la calidad del producto informativo resultante.

Algunos de estos instrumentos de accountability gozan de una larga tradición, mientras que otros mecanismos se han incorporado de forma más reciente (Alsius, RodríguezMartínez y Mauri-Ríos, 2018). Puesto que los instrumentos de rendición de cuentas permiten sustituir la intervención reguladora y política en aras de preservar la independencia y pluralismo de los medios de comunicación, resulta de especial relevancia sistematizar y categorizar dichos instrumentos. Además, dado que la "rendición de cuentas es una cuestión que preocupa tanto a los periodistas como a las audiencias" (Chaparro-Domínguez, Suárez-Villegas y Rodríguez-Martínez, 2019, p.2), es imprescindible ahondar en los mecanismos que facilitan la participación de los usuarios, así como determinar de qué forma los ciudadanos perciben estos instrumentos.

\section{OBJETIVOS Y METODOLOGÍA}

Con el propósito de ahondar en la participación de la audiencia en la rendición de cuentas de los medios, la presente investigación presenta tres objetivos específicos: sistematizar y clasificar los instrumentos de accountability que permiten fomentar la transparencia, la autorregulación y la participación de los usuarios (OE1); examinar los instrumentos que facilitan la participación (OE2); y analizar la percepción de los ciudadanos españoles sobre los instrumentos de accountability que facilitan la participación (OE3). 
Para llevar a cabo estos objetivos, se han aplicado varias técnicas de investigación complementarias. En primer lugar, con el objetivo de conocer y sistematizar los sistemas de rendición de cuentas que ahondan en las tres dimensiones clave de la accountability (OE1), se ha empleado una metodología fundamentada en el sistema de muestreo no probabilístico conocida como snowball sampling o bola de nieve. Esta estrategia, empleada principalmente en el marco de investigaciones cualitativas (Brickman-Bhutta, 2012; Bryman, 2016), está indicada para estudios donde a priori se desconoce la muestra a examinar. Su aplicación se "fundamenta en ir identificando sujetos -o experiencias- que se van incorporando progresivamente a la muestra" (Mauri-Ríos y Ramon-Vegas, 2015, p.381).

Se partió de un listado inicial de instrumentos detectados por Fengler et al. (2014) en el proyecto MediaAcT (Media Accountability and Transparency in Europe). Durante el período comprendido entre 2014 y 2019, a lo largo de dos proyectos de investigación competitivos financiados por el Ministerio de Economía y Competitividad (Transparencia y Rendimiento de cuentas en la Información Periodística, CSO201239138; Accountability y Culturas Periodísticas en España. Impacto y propuesta de buenas prácticas en los medios de comunicación españoles, CSO2015-66404-P), se han aplicado varias técnicas de investigación -análisis del contenido de sitios web de medios nacionales e internacionales, entrevistas en profundidad con expertos y grupos de discusión con ciudadanos- que han permitido recopilar múltiples casos de buenas prácticas en materia de accountability. Algunos de estos instrumentos de rendición de cuentas siguen vigentes en la actualidad, otros han cesado su funcionamiento o se han reconfigurado a lo largo del tiempo.

En segundo lugar, una vez clasificados los instrumentos de accountability, se procedió a examinar los mecanismos que en particular facilitan la participación de los usuarios (OE2). Para realizar dicha aproximación, se empleó la técnica del análisis del contenido cualitativo (Bryman, 2016). Partiendo del documento Best Practice Guidebook del proyecto MediaAcT (Bichler et al., 2012), se construyó un sistema de codificación que pivotó sobre las siguientes categorías: producción interna o externa a los medios; 
descripción de las características del instrumento; y valoración del mecanismo para la rendición de cuentas.

En tercera y última instancia, se aplicó la técnica del focus group para obtener una radiografía sobre el conocimiento que los ciudadanos tienen de los instrumentos de accountability que fomentan la participación (OE3). Entre el 12 abril y el 31 de mayo de 2018 se celebraron un total de 6 grupos de discusión en diferentes ciudades españolas: Barcelona (GD-01), Castellón (GD-02), Madrid (GD-03), Sevilla (GD-04), Mondragón (GD05) y Santiago de Compostela (GD-06), con 38 participantes en total. De todos los participantes, $58 \%$ fueron mujeres $(n=22)$ y el $42 \%$ fueron hombres $(n=16)$. Para asegurar la confidencialidad en la presentación de los datos, las citas se presentan con el identificador del grupo de discusión y el género de cada participante (H: Hombre; $M$ : Mujer).

\section{RESULTADOS}

\subsection{SISTEMATIZACIÓN Y CLASIFICACIÓN DE LOS INSTRUMENTOS DE RENDICIÓN DE CUENTAS.}

A partir de la exploración realizada, se han detectado un total de 17 tipos de instrumentos de rendición de cuentas producidos en el sí de las empresas periodísticas (Tabla 1). Fomentando la transparencia, encontramos blogs de la redacción donde se explican los procesos de creación de las noticias. También se observan páginas con información corporativa y otros elementos que contribuyen a fomentar la transparencia corporativa. En la dimensión que fomenta la autorregulación se observan defensores del lector o de la audiencia, ya sean con formato tradicional (Maciá-Barber, 2006) o actuando en línea. En este último caso, son habituales los blogs del defensor en línea donde se expone las decisiones o debates realizados (Ramon-Vegas, Billings y RojasTorrijos, 2019). También existen blogs de periodistas incluidos en la página web del medio. Otro paquete de instrumentos relevantes son los libros de estilo, códigos éticos (generales o temáticos) de los propios medios o estatutos de redacción que cuentan con indicaciones deontológicas (Alsius, 1999). 
Tabla 1. Instrumentos de accountability impulsados por los medios, según su dimensión: transparencia, autorregulación o participación.

\begin{tabular}{|c|c|}
\hline Dimensión & $\begin{array}{l}\text { Instrumentos de rendición de cuentas impulsados por los } \\
\text { medios }\end{array}$ \\
\hline Transparencia & $\begin{array}{l}\text { Blogs editoriales/ blogs de la redacción } \\
\text { Páginas con información corporativa en las webs de los medios } \\
\text { Elementos de transparencia informativa }\end{array}$ \\
\hline Autorregulación & $\begin{array}{l}\text { Defensores del lector/audiencia } \\
\text { Blogs en línea del defensor del lector (ombudsman online) } \\
\text { Blogs de periodistas incluidos en la página web del medio } \\
\text { Libros de estilo/ códigos éticos generales de los medios/ código } \\
\text { temático interno/ Estatuto de redacción } \\
\text { Crítica del periodismo en secciones o espacios sobre medios } \\
\text { Comités profesionales }\end{array}$ \\
\hline $\begin{array}{l}\text { Participación de los } \\
\text { usuarios }\end{array}$ & $\begin{array}{l}\text { Cartas al director/llamadas/SMS/WhatsApp } \\
\text { Comentarios de los usuarios y valoración de noticias en la web } \\
\text { Comentarios de los usuarios en las redes sociales. Rendición de } \\
\text { cuentas a través de los canales generales del medio en las redes } \\
\text { sociales o a través de cuentas específicas creadas por el medio } \\
\text { para este propósito } \\
\text { Botones de corrección de errores } \\
\text { Chats y encuentros digitales con los lectores } \\
\text { Contribuciones de los usuarios en la creación y revisión de los } \\
\text { contenidos (I). Posibilidad de los lectores de realizar sus } \\
\text { aportaciones, sugerir enfoques y ayudar en la búsqueda de } \\
\text { fuentes } \\
\text { Contribuciones de los usuarios en la creación y revisión de los } \\
\text { contenidos (II). Existencia de canales dedicados a las } \\
\text { contribuciones de los lectores } \\
\text { Servicio de quejas/reclamaciones }\end{array}$ \\
\hline
\end{tabular}

Dentro de la dimensión de la participación es donde se encuentran más instrumentos. Desde las tradicionales cartas al director, que han evolucionado a llamadas, SMS o mensajes de WhatsApp; a servicios de quejas, comentarios de los usuarios en noticias publicadas a través de la web o de las redes sociales o a través de cuentas específicas creadas por los medios o chats y encuentros digitales con el público (Craft, Vos y Wolfgang, 2016). También encontramos instrumentos más sofisticados como botones de corrección de noticias (Karlsson, Clerwall y Nord, 2017), o instrumentos para que los usuarios puedan ayudar a crear y revisar contenidos, ya sea realizando aportaciones 
(fuentes, enfoques, temáticas) o a través de canales creados de forma específica para esta participación activa.

La exploración realizada también ha permitido detectar 17 tipologías de instrumentos impulsados de forma externa a los medios (Tabla 2). Dentro de la dimensión de la transparencia se encuentran observatorios de medios que creados desde ámbitos distintos (universidades, entes autorreguladores, organizaciones independientes) permiten analizar el desarrollo informativo de los medios ajustado a parámetros deontológicos (Rosique-Cedillo y Barranquero-Carretero, 2016). También, y con propósito similar, se detectan revistas y publicaciones dedicadas a la crítica de medios, estudios de mercado, estudios de opinión, publicaciones especializadas o análisis realizadas desde la academia.

En la dimensión de autorregulación se encuentran algunos de los instrumentos con más tradición como los consejos de prensa o audiovisuales o los códigos éticos externos (creados generalmente por instituciones de autorregulación) o recomendaciones. También juegan un papel relevante en la autorregulación los colegios profesionales, sindicatos de periodistas o asociaciones de la prensa (Rodríguez-Martínez, López-Meri, Merino-Arribas, y Mauri-Ríos, 2017), así como la formación de los periodistas. Finalmente, se observa que también ejercen un rol de autorregulación las webs de instituciones y asociaciones profesionales.

La dimensión de participación es donde nuevamente se detectan más instrumentos. Algunos de tradicionales como las asociaciones de espectadores. Pero la mayoría son de creación más reciente aprovechando las potencialidades de internet y las redes sociales. Así, encontramos desde blogs de ciudadanos, comunicadores o académicos que participan en la observación crítica de los medios o cuentas particulares en las redes sociales con este mismo objetivo. Hay otros instrumentos impulsados por la ciudadanía como sistemas de notificación de errores, sistemas de envío de información de interés público a los medios o páginas web de asociaciones de espectadores y usuarios de los medios. 
Tabla 2. Instrumentos de accountability impulsados de forma externa a los medios, según su dimensión: transparencia, autorregulación o participación.

\begin{tabular}{|c|c|}
\hline Dimensión & $\begin{array}{l}\text { Instrumentos de rendición de cuentas impulsados por los } \\
\text { medios }\end{array}$ \\
\hline Transparencia & $\begin{array}{l}\text { Observatorios de medios } \\
\text { Revistas y publicaciones dedicadas a la crítica de medios } \\
\text { Estudios de mercado } \\
\text { Publicaciones especializadas en medios } \\
\text { Academia } \\
\text { Estudios de opinión }\end{array}$ \\
\hline Autorregulación & $\begin{array}{l}\text { Colegios/sindicatos/asociaciones de periodistas } \\
\text { Consejos de prensa y consejos audiovisuales } \\
\text { Códigos éticos externos y recomendaciones } \\
\text { Formación } \\
\text { Webs de instituciones y asociaciones profesionales }\end{array}$ \\
\hline $\begin{array}{l}\text { Participación de los } \\
\text { usuarios }\end{array}$ & $\begin{array}{l}\text { Asociaciones de espectadores } \\
\text { Blogs de ciudadanos, comunicadores y/o académicos } \\
\text { Iniciativas particulares: redes sociales de crítica de medios } \\
\text { Sistemas de notificación de errores impulsados por los } \\
\text { ciudadanos } \\
\text { Sistemas de envío de información confidencial para su uso por } \\
\text { parte de los medios } \\
\text { Páginas web de asociaciones de espectadores y usuarios de los } \\
\text { medios }\end{array}$ \\
\hline
\end{tabular}

\subsection{ANÁLISIS DE LOS INSTRUMENTOS QUE FOMENTEN LA PARTICIPACIÓN}

En la dimensión de participación, si se contemplan los instrumentos impulsados dentro y fuera de los medios, se detectan un total de 12 tipologías de instrumentos de rendición de cuentas. A continuación, se analizan a partir de ejemplos relevantes presentes en el ámbito mediático español e internacional.

\subsubsection{Cartas al director/llamadas/SMS/WhatsApp}

Se trata de una tipología de instrumentos tradicionales, al inicio unidireccionales, que han ido evolucionando en la medida que han ido apareciendo nuevas formas de comunicación y mensajería instantánea que permiten, si el medio lo quiere, aplicar formas de bidireccionalidad. Todos los periódicos en papel incorporan el apartado de cartas al director, que acostumbra a tener también espacio en la web. Pero estas 
fórmulas se han ido sofisticando con la incorporación de sistemas como los SMS, formularios web o el WhatsApp. Algunos ejemplos relevantes los encontramos en medios de referencia como The New York Times (https://www.nytimes.com/section/opinion/letters), The Guardian (https://www.theguardian.com/info/2015/aug/12/whatsapp-sharing-stories-with-theguardian) o CNN (https://edition.cnn.com/feedback).

Figura 1. Sección de cartas al director de The New York Times.

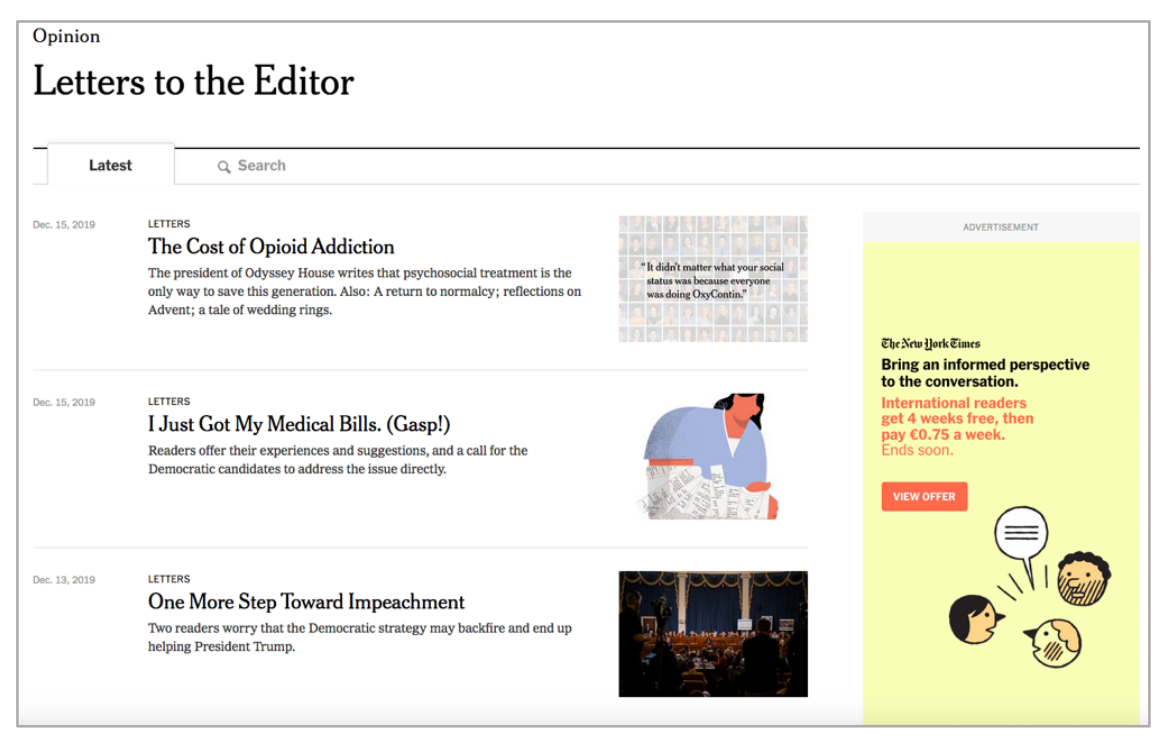

\subsubsection{Comentarios de los usuarios en la web y en redes sociales}

Uno de los aspectos más relevantes que aportó internet fue la capacidad de interacción y participación en tiempo real de los usuarios con los medios de comunicación. Las redes sociales no son solamente un canal de difusión, también ejercen de instrumento de rendición de cuentas ya que a través de estas redes los ciudadanos pueden monitorizar la calidad de los medios y expresar comentarios, críticas y sugerencias (Mauri-Ríos y Ramon-Vegas, 2015). Algunos medios, como El Periódico de Catalunya, han creado cuentas específicas en Twitter para recoger la opinión de los lectores (https://twitter.com/epentretodos). El periódico digital catalán VilaWeb también ha creado una red social propia solo para suscritores donde pueden comentar noticias y expresar opiniones. 
Figura 2. Cuenta de Twitter @EPentretodos, creada por El Periódico de Catalunya.

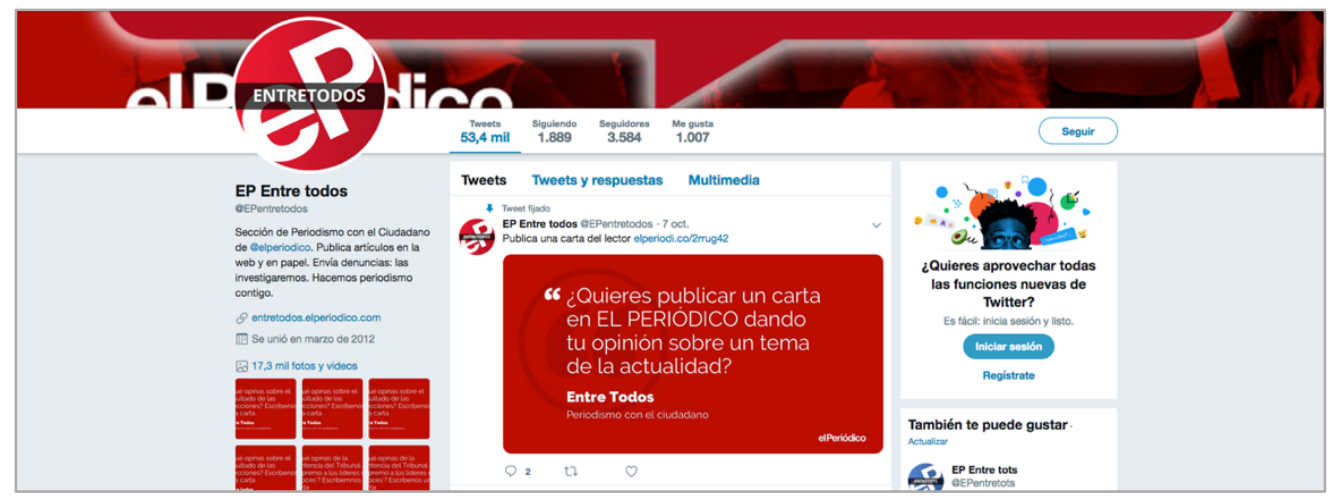

Estas opiniones, denuncias o sugerencias no se expresan solamente a través de las redes sociales, sino también en las páginas web de los propios medios que habilitan opciones de comentarios. En los últimos años, han sido muchos los medios de comunicación que han limitado, moderado o han generado registros para estos comentarios para evitar malas praxis que se estaban cometiendo. Un estudio de Diaz Noci et al. (2012) analizó más de 35.000 comentarios emitidos en 7 periódicos catalanes llegando a la conclusión que en su mayoría no contribuían a fortalecer un debate democrático. Para evitar estas situaciones, medios como Eldiario.es han decido limitar el número de comentarios por día para evitar spam y malas prácticas, detallando una política de participación que recoge las características que la audiencia debe seguir (https://www.eldiario.es/participación/).

\subsubsection{Botones de corrección de errores}

Algunos medios, como The Washington Post, fueron pioneros en 2011 en introducir en sus páginas web aplicaciones que servían para que los lectores pudieran informar sobre errores que hubieran detectado. Actualmente, este servicio sigue activo, pero se realiza mayoritariamente a través de correo electrónico. Medios internacionales como HuffingtonPost (https://www.huffpost.com/static/contact-us), The New York Times (https://help.nytimes.com/hc/en-us/articles/115015385887-Contact-Us) o The Texas Tribune (https://www.texastribune.org/corrections/) optan por este mismo sistema. Otras organizaciones, como El Tiempo en Colombia (https://www.eltiempo.com) o De Staandard en Bélgica (https://www.standaard.be) incluyen en cada una de las noticias 
publicadas botones de corrección, que permiten a los usuarios rellenar un formulario con sus datos y la explicación de los errores que han detectado.

Figura 3. Sistema de notificación de errores en El Tiempo.

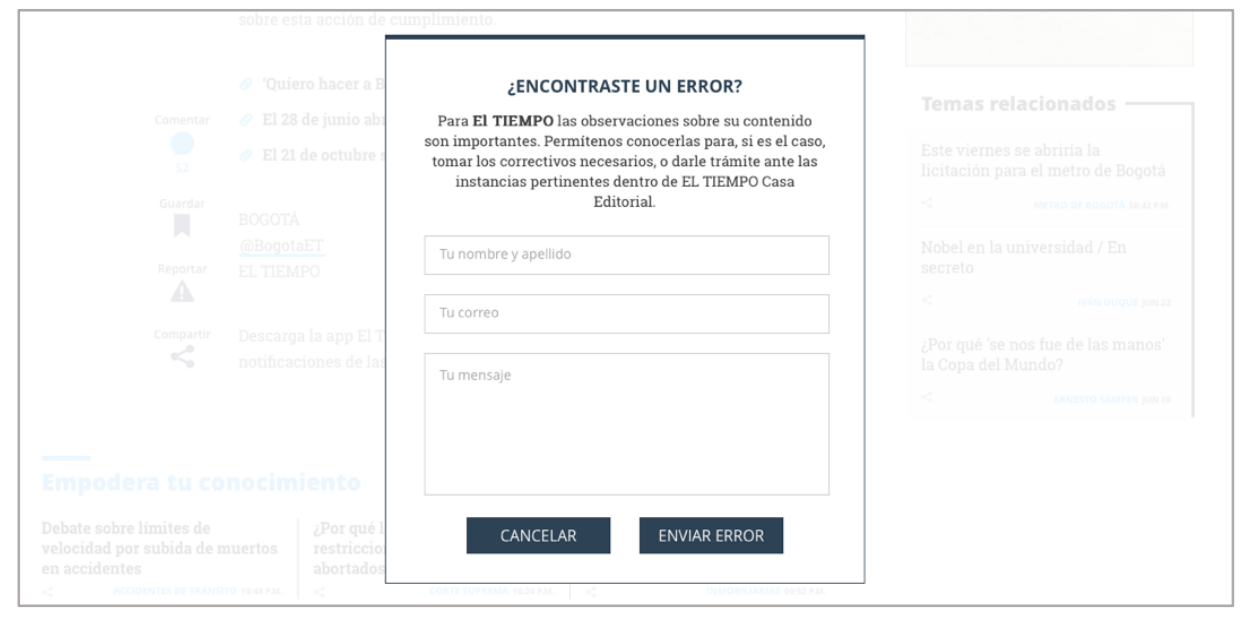

\subsubsection{Chats o videochats con los lectores}

Son distintos los medios de comunicación que han creado chats (en formato escrito) o videochats (formato audiovisual) para fomentar la interacción en tiempo real entre los lectores, el equipo editorial y expertos. Estos encuentros sirven para promover la participación de los usuarios, pero son a la vez un ejercicio de transparencia del medio de comunicación. Algunos medios pioneros fueron The Washington Post con los chats Live Q\&A (Questions \& Anwers) (Mauri-Ríos y Ramon-Vegas, 2015). Como ha sucedido en otros medios, actualmente este chat ya no está operativo. Es lo mismo que ocurrió con el vídeochat con el editor que el periódico Ara impulsó en 2014 y que no ha tenido continuidad. Algunos medios, como el Ideal de Granada, han ido más allá y han creado encuentros offline con los lectores para que puedan conocer a los periodistas y preguntar sobre el trabajo que realizan (https://www.ideal.es/promociones/actualidadgranada-rafael-20191115095037-nt.html). 
Figura 4. Encuentros de Ideal entre suscriptores y periodistas.
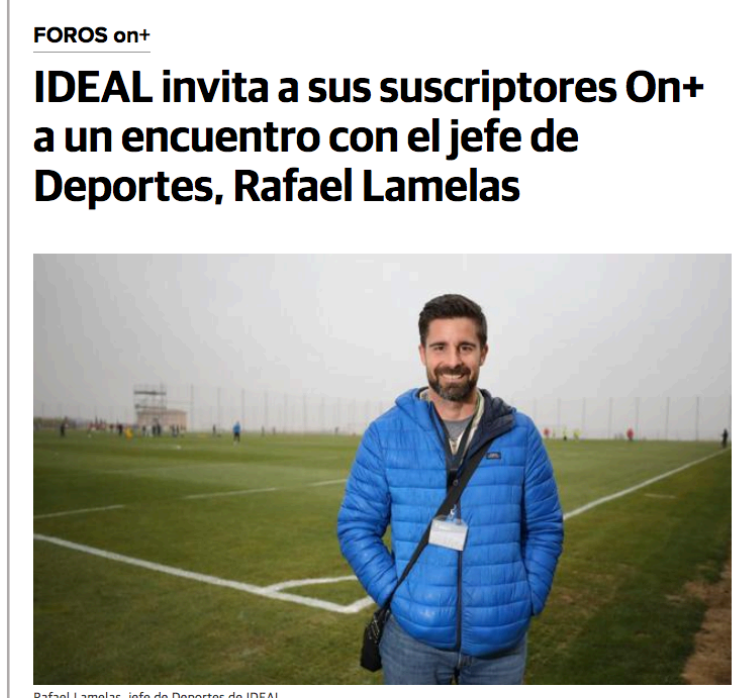

El periódico da la posibilidad a sus suscriptores de conocer de cerca a los periodistas detrás de las noticias

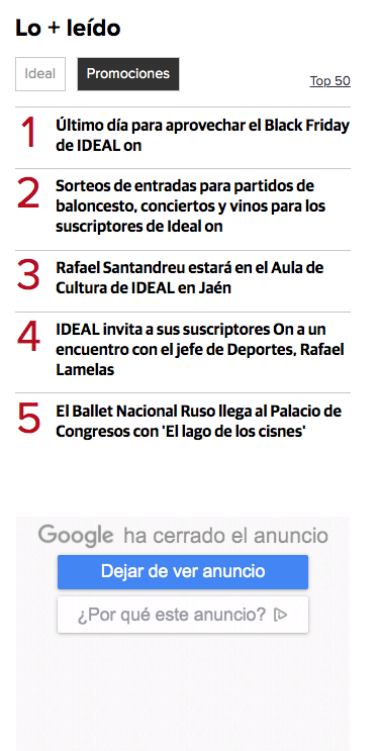

\subsubsection{Contribución de los usuarios en la creación y revisión de contenidos}

Vilaweb envía cada noche en exclusiva a sus suscritores, que conforman una comunidad de lectores, el editorial que al día siguiente publica el director, Vicent Partal, para que puedan hacer llegar comentarios que se añaden al final del texto escrito por el director (https://www.vilaweb.cat/registre/registre-subscriptor). En The New York Times ponen a disposición de los lectores un apartado para que puedan enviar comentarios de opinión, ya sean por escrito o en vídeo (https://help.nytimes.com/hc/enus/articles/115014925188-How-to-Submit-an-Op-Doc). La Marea dispone desde hace años de una sección llamada "Los socios/as escriben" para fomentar la creación de contenidos de los usuarios (http://www.lamarea.com/secciones/los-socios-escriben). El apartado "Entre Todos" de El Periódico de Cataluña incluye un "Buzón de denuncias ciudadanas" que tiene por objetivo que los lectores puedan realizar quejas sobre incumplimientos de la administración pública y, a partir de ahí, los periodistas investigan esa denuncia para buscar respuestas (https://www.elperiodico.com/es/entretodos/tema/buzon-denuncias-ciudadanas-3994).

\subsubsection{Servicio de quejas/reclamaciones}


Se trata de apartados donde los usuarios pueden hacer llegar al medio de comunicación aquellas quejas o reclamaciones por cuestiones sobre el tratamiento informativo o las opiniones versadas sobre los temas publicados. En periódicos com The New York Times encontramos apartados en la web para que los lectores puedan formular quejas o reclamaciones. En este caso, se trata de un buscador que permite acotar la cuestión sobre la que se quiere formular la petición (https://help.nytimes.com/hc/en-us). Otro periódico como Le Monde pone a disposición dos correos electrónicos para que los lectores puedan reportar quejas sobre el tratamiento general de una información.

\subsubsection{Asociaciones de espectadores y Páginas web de asociaciones de espectadores}

En el ámbito de la participación y externa a los medios, destacan las asociaciones de espectadores, instrumentos con una larga tradición que tienen como objetivo velar por una comunicación responsable y de calidad y proteger los derechos de los ciudadanos (Alsius et al., 2018). En España existen distintas asociaciones, pero la más importante en la Asociación de Usuarios de la Comunicación (http://www.auc.es/), que tiene tiene por misión defender los intereses de la ciudadanía ante los medios. En Cataluña, el Consell de l'Audiovisual (ente regulador del sistema audiovisual catalán) creó en 2001 el Foro de entidades de personas usuarias del audiovisual (https://www.cac.cat/forum-entitats) con el fin de ser un espacio de intercambio de ideas y conocimientos entre instituciones de la sociedad civil para promover un sistema mediático de calidad. A nivel internacional destaca el MediaWatch en Reino Unido, una institución creada en 1965 que se autodefine como grupo de presión para velar por un entorno mediático de calidad y respetuoso con los derechos de los menores y las familias (https://mediawatchuk.com/). 
Figura 5. Portal web de MediaWatch.

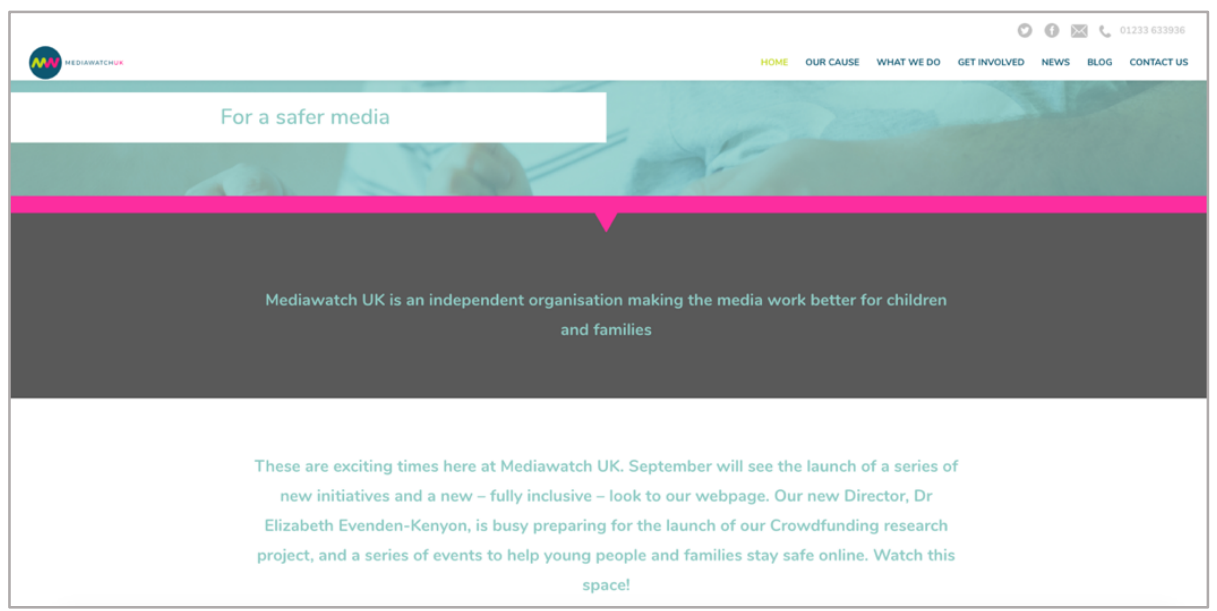

\subsubsection{Iniciativas particulares: redes sociales de crítica de medios}

En los últimos años, Facebook y muy particularmente Twitter se han convertido en espacios de intercambio de opiniones, comentarios y críticas hacia el comportamiento de los medios de comunicación. La necesidad de los medios de tener una ventana abierta en las redes les obliga a dar respuesta a esta crítica constante (Mauri-Ríos y Ramon-Vegas, 2015).

\subsubsection{Blogs de ciudadanos, comunicadores y/o académicos}

Desde una mirada crítica hacia los medios, y con el fin de promover un sistema mediático responsable, han aparecido en los últimos años blogs impulsados por comunicadores, académicos y ciudadanos. Algunos ejemplos relevantes son La Buena Prensa (http://labuenaprensa.blogspot.com), realizado por Miguel Ángel Jimeno, profesor de la Universidad de Navarra; La Mala Prensa (http://www.malaprensa.com), editado por Josu Mezo; Papers Papers (http://www.paperpapers.net), a cargo de Gonzalo Peltzer y Toni Piqué; o Periodismo Deportivo de Calidad, del profesor de la Universidad de Sevilla José Luis Rojas Torrijos (https://periodismodeportivodecalidad.blogspot.com). 
Figura 6. Blog de crítica de medios "Malaprensa".

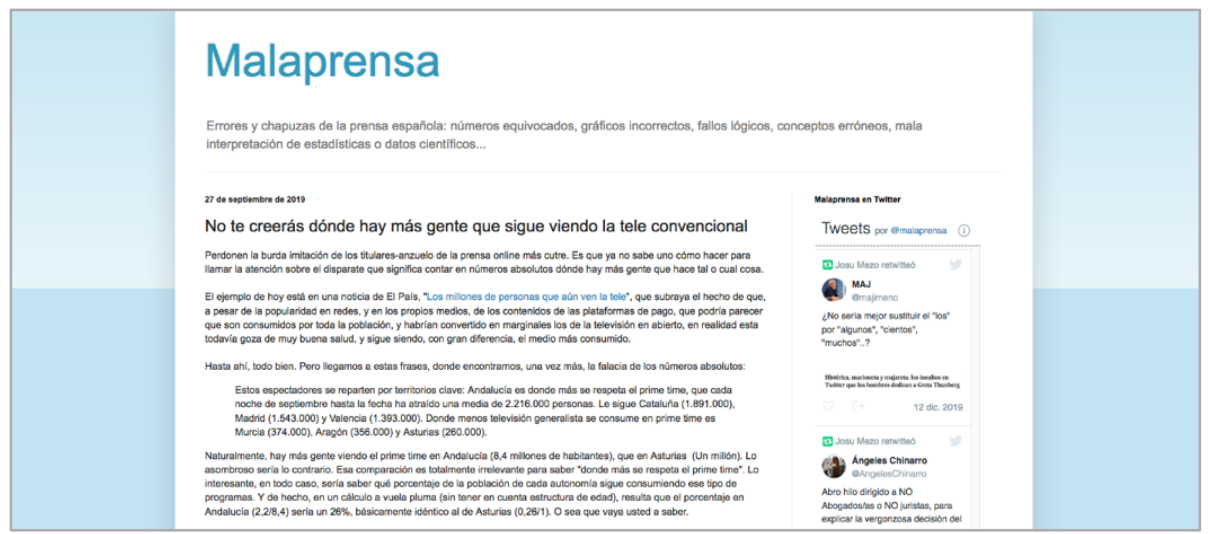

\subsubsection{Sistemas de notificación de errores impulsados por los ciudadanos}

Existen otras iniciativas impulsadas de forma independiente por ciudadanos que fomentan la rendición de cuentas a partir de la detección y notificación de errores que se hayan publicado en los medios. Una de las primeras plataformas a nivel internacional en realizar este tipo de acciones fue MediaBugs (http://mediabugs.org/). Aunque la página web sigue siendo consultable, desde hace 7 años no han realizado ninguna actualización. En España, apareció un instrumento basado en este mismo sistema de notificación de errores de medios, Fixmedia.org, que tampoco está ya operativo.

3.2.11. Sistemas de envío de información de interés público para su posterior uso por parte de los medios

En los últimos años, varios medios de comunicación han implantado sistemas que permiten a los usuarios mandar de forma segura material confidencial, con el objetivo de contribuir al posterior desarrollo de investigaciones periodísticas. En el ámbito internacional, medios como The New York Times aceptan el envío de material por WhatsApp, Signal, correo electrónico, correo postal o SecureDrop (https://securedrop.org). Este último es un sistema que también utilizan otros medios de referencia como The Washington Post o The Guardian. En España, el sistema equivalente es Filtrala (https://filtrala.org), operado por la Associated Whistleblowing Press (AWP) y empleado por medios y organizaciones independientes como Eldiario.es, Mongolia, porCausa, CIVIO o Facua. 
Figura 7. "Fíltrala", plataforma de filtraciones seguras y anónimas.

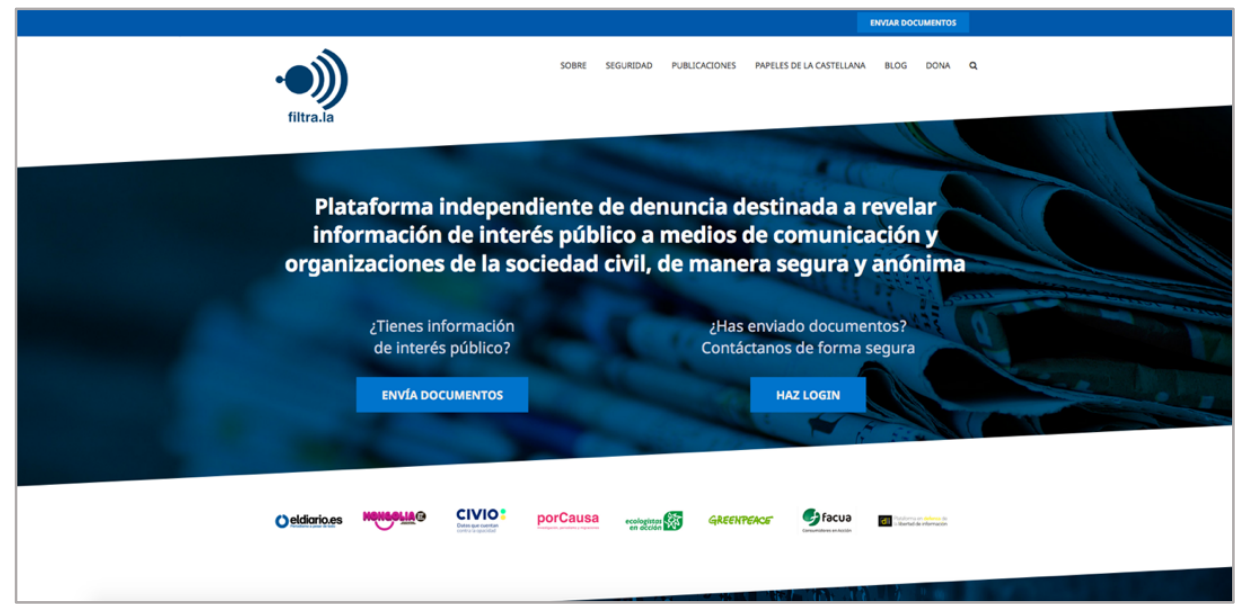

\subsection{PERCEPCIÓN DE LA CIUDADANÍA SOBRE LOS INSTRUMENTOS QUE FOMENTAN LA} PARTICIPACIÓN

A través de los 6 grupos de discusión realizados, se puede observar la opinión que los ciudadanos tienen acerca de aquellos instrumentos de rendición de cuentas que fomentan la participación del público. Hay algunos instrumentos descritos en el apartado anterior que son desconocidos o no valorados, como por ejemplo los servicios de quejas. A continuación, se detallan las opiniones y observaciones del resto de instrumentos que sí que eran conocidos y fueron debatidos en los grupos de discusión.

\subsubsection{Cartas al director}

Se trata de un instrumento que la mayoría de las personas que conformaron los grupos de discusión han utilizado alguna vez y han tenido una experiencia positiva. A diferencia de los comentarios en las noticias o en las redes sociales, las cartas al director se consideran "más reposadas, más reflexionadas" (GD01-M) y, por tanto, más útiles o valiosas. Apuntan que una carta "requiere pensarla, estructurarla" por lo que es "bastante más útil que un comentario que tú haces en internet en un momento dado, visceral" (GD01-M). También hay quien piensa que las opiniones de los lectores no tienen porqué ser especialmente interesantes (GD01- H) pero en general este instrumento es bien valorado. 


\subsubsection{Comentarios de los usuarios}

Los participantes de los grupos focales valoran como positivos los instrumentos habilitados en los medios para poder comentar las noticias. De hecho, la mayoría de ellos explica que a menudo ellos también utilizan esta herramienta. Consideran que son espacios que pueden tenir distintas funciones como crear contenidos, realizar denuncias de temas, aportar nueva información, incentivar el debate entre la audiencia, reflexionar e incluso contribuir al cambio de opinión sobre una noticia. Para algunos es el espacio más democrático en el ámbito periodístico, un "punto de encuentro" (GD06H). Los ciudadanos consideran que es un "espacio colaborativo" que permite una mayor participación al lector (GD01-H) sobretodo cuando se intenta debatir "más razonadamente" (GD02-H). Pese a la buena valoración de este instrumento sí que existen algunas lagunas. Por ejemplo, se "desconfía" que los periodistas se lean estos apartados y se sospecha que estos espacios participados son más bien una manera de "mover" la noticia, de generar visitas, que no de interés por la opinión de la audiencia" (GD01-M). Otra consideración negativa es que muchos de los comentarios aportan poco o nada a la reflexión por lo que a menudo se consideran espacios de opinión sin argumentos, y a veces ofensivos o inadecuados, "con muchas faltas de respeto" (GD02M) por lo que a algunos ciudadanos no les parece el major instrumento para "mejorar los medios" (GD02-M). Una frase sintetiza este sentimiento contradictorio: "Me parece útil y a la vez peligroso" (GD04-M).

A diferencia de los comentarios en las noticias publicadas en las páginas web, que en general se veían como positivas, se asocia a los comentarios sobre medios en las redes sociales con aspectos negativos. Un ciudadano lo resumía diciendo que "en las redes está todo el mundo en contra de todo" (GD04-H). Aunque también hay aspectos positivos. Por ejemplo, se destaca que en las redes se genera una mayor interrelación, tanto entre usuarios como con medios y periodistas porque estos últimos se sienten más interpelados. También se valora que en las redes se aporta y comparte nueva información entre los usuarios que puede ayudar a "profundizar" en los temas (GD04M). La monitorización en rendición de cuentas a través de las redes también es un aspecto a tener en cuenta ya que es más fácil "dejar rápidamente en evidencia a un 
medio que calla ante una acusación o una crítica" (GD06-M). En general se considera que los medios, cuando reciben críticas en las redes sociales, se asustan y quieren reaccionar y se ven obligados a "pedir disculpas haciendo un comunicado" (GD06-M).

\subsubsection{Botones de corrección de errores}

Los botones de corrección de errores son vistos de forma "útil" (GD06-M) y positiva entre las personas de los grupos focales, aunque es un instrumento bastante desconocido. Algunos participantes consideran que la corrección de errores "se debería facilitar más" (GD01-M). Esta transparencia en la corrección puede generar un rechazo o desconfianza hacia el medio, que expone sus faltas o errores, y prefieren medios "que se informen bien" antes de publicar (GD04-M). Para otras personas, esta exposición de errores genera justamente el efecto contrario, confianza en el medio: "si son así de decentes igual los vuelvo a leer un poquito" (GD06-M).

\subsubsection{Chats y encuentros digitales con los lectores}

Los chats o encuentros digitales con lectores son poco conocidos. La mayoría de los participantes en los grupos de discusión no han utilizado nunca este instrumento. Aquellos que han participado en alguno reconocen que son interesantes, aunque consideran que son de carácter más lúdico que crítico o reflexivo: "normalmente no encuentras mucha profundidad" (GD06-H).

\subsubsection{Creación y revisión de contenidos}

La participación de la audiencia en la producción de noticias se visualiza, en general, como un instrumento positivo, "fundamental y extraordinario" (GDO3-H) y cada vez más habitual, gracias a la facilidad de la población para aportar información grabada con el teléfono móvil. Eso provoca que la participación de la audiencia en la producción sea "cada vez es mayor" aunque eso no quiera decir que haya una mayor "corrección de las noticias" (GD01-H). En todo caso, se constata que en muchas ocasiones las noticias "se basan en la recogida de vídeos o de imágenes del público" aunque luego los periodistas deben "dar contraste en la medida de lo posible" (GD01-M). De hecho, se apunta como preocupación el hecho que desde el periodismo no se haga "crítica" a estos datos 
aportados por los ciudadanos (GD03-M). También se comenta que esta participación del público en el contenido de las noticias está cambiando la manera de hacer periodismo. Algunas personas destacan la mayor veracidad de estas aportaciones que las del propio periodista gracias al "fact check que hacen los lectores que están conectados a internet, y es gracias a ello que muchas veces (...) se generan noticias o se sigue el hilo conductor para investigar una cosa" (GD03-M). No todos los participantes lo ven igual. Algunos de éllos dudan de si esas informaciones son "veraces" (GD05-M) y señalan que en todo caso se debe "filtrar" bien los datos aportados (GD06-H).

\subsubsection{Asociaciones de espectadores}

Las asociaciones de espectadores son muy desconocidas. Ninguna de las personas participantes ha tenido relación con una de estas entidades ni conocen ejemplos de ellas. La opinión sobre estos organismos es positiva aunque el hecho que sean tan poco conocidas genera incertidumbre: "¿lo han puesto en alguna televisión, ha salido en algún periódico en alguna página que diga que te puedes asociar?" (GD01-H). Se apuntan algunas soluciones para dar más a conocer este instrumento. Por ejemplo, que hubiera "una casilla, un recuadrito cada día en el periódico" (GD01-H) para que la gente supiera de su existencia.

\subsubsection{Iniciativas particulares}

Las redes sociales pueden ser un espacio muy útil por su enorme repercusión y visibilidad entre los consumidores de medios. Una crítica en las redes se considera en ocasiones más capaz de movilizar consciencias o cambiar actitudes que las entidades corporativas. Además, se apunta a que, a diferencia de otros ámbitos sociales donde es mejor actuar en colectivo, "con el periodismo es más eficaz la actuación individual, la crítica individual, el activismo individual, del consumidor, del ciudadano" (GD06-M) a través de las redes sociales. A menudo, no se busca tanto el feedback con el medio sino respuestas "de otros usuarios que están a favor de lo que estás comentando. Entonces se generan retweets, se generan contestaciones" (GD03-M) y eso se considera que genera más presión para el medio en cuestión. También están los que consideran que la crítica en las redes, como pasa con otros instrumentos de participación, sólo sirve 
para que la gente de su opinión: "tú descansas, te desahogas pero no va a más" (GD02M).

La respuesta que hacen los medios a las quejas de la audiencia que se producen en las redes sociales es un método de rendición de cuentas que se está generalizando rápidamente. Según algunas personas, sin embargo, se responde dependiendo de en qué medida la queja ha generado interés o se ha replicado en las redes. "Hasta que no se ven perjudicados por el número de personas que se han quejado es muy grande, no van a reaccionar (...) entonces sí que dicen perdón porque les interesa mantener una buena imagen para atraer a lectores" (GD01-M).

\subsubsection{Blogs de ciudadanos, comunicadores y/o académicos}

Se trata de un instrumento nuevamente no muy conocido entre los participantes. Aquellos que sí que sabían de su existencia, los circumscriben a blogs de periodistas (no se mencionan blogs de ciudadanos ni de académicos) y los consideran positivos. Son definidos como espacios de crítica del periodismo donde "se expresa mucha información" (GD02-H) con capacidad para profundizar en los entresijos de la información: "ves como una noticia se puede tergiversar con los mismos datos, como te lo pueden contar como si fuera una tendencia o como si fuera un caso puntual" (GD03M).

\section{CONCLUSIONES}

La investigación revela la aparición de un amplio abanico de instrumentos de rendición de cuentas -impulsados de forma interna y externa a los medios- en el ámbito español e internacional que ahondan en la transparencia, la autorregulación y la participación de los usuarios en el proceso de creación y revisión de los contenidos periodísticos. En este último ámbito, a aquellos mecanismos de accountability tradicionales y con una sólida implantación como las cartas al director se les han sumado en los últimos años el desarrollo de nuevas posibilidades online como los comentarios en las noticias y en las redes sociales, los botones de corrección de errores o los blogs de crítica de medios. El 
desarrollo de instrumentos en línea no ha imposibilitado la experimentación con nuevos espacios de relación presencial con las audiencias.

Como señalan Suárez-Villegas, Rodríguez-Martínez, Mauri-Ríos y López-Meri (2017, p.323), todos estos instrumentos "comparten la conciencia de que los medios son decisivos en la construcción social del imaginario colectivo". Empleando estos mecanismos, los usuarios pueden proporcionar un escrutinio continuo de la información periodística y manifestar sus pensamientos, valores y expectativas respecto al contenido y sus formas de producción. De este modo, las audiencias pueden jugar un papel transformativo en el periodismo contemporáneo, corresponsabilizándose de la actividad de rendir cuentas.

Más allá de sistematizar y categorizar estos instrumentos, este trabajo ha ahondado en el conocimiento y percepción que los ciudadanos españoles tienen sobre los mecanismos de accountability que fomentan la participación. Las aportaciones recogidas en los grupos de discusión, donde se evidencia desconocimiento de buena parte de estos instrumentos de rendición de cuentas que fomentan esta participación ciudadana, señalan que queda un largo camino por recorrer. Pese a que España es uno de los países que forman parte del modelo Mediterráneo o Pluralista Polarizado descrito por Hallin y Mancini (2004) que presenta un espectro más amplio de instrumentos de rendición de cuentas impulsados de forma interna y externa a los medios (Alsius et al., 2018), muchos de estos instrumentos son aún invisibles a los ojos de la ciudadanía. Frente a mecanismos ampliamente utilizados y valorados positivamente como las cartas al director o los comentarios en las noticias publicadas en la web de los medios, otros como las asociaciones de espectadores, los botones de corrección de errores, los blogs de crítica o los chats o encuentros digitales- son poco conocidos y, por consiguiente, poco utilizados. Algunos -como el servicio de quejas o los sistemas de notificación de errores impulsados por los propios ciudadanos- directamente son desconocidos por la audiencia.

A la luz de estos hallazgos, resulta imprescindible que empresas periodísticas y organizaciones independientes tomen consciencia de la necesidad de revisar y actualizar 
su abanico de instrumentos de rendición de cuentas, potenciando su visibilidad e incentivando que los usuarios los empleen de forma activa para monitorizar la calidad del trabajo periodístico. Desde el ámbito académico, futuras investigaciones deberán seguir examinando el surgimiento, la consolidación y la reconfiguración de los instrumentos de accountability que fomentan la participación, además de trazar la evolución de otros mecanismos tradicionales e innovadores que ahondan en la transparencia y la autorregulación.

\section{REFERENCIAS BIBLIOGRÁFICAS}

Alsius, S. (1999). Codis ètics del periodisme televisiu. Barcelona: Pòrtic.

Alsius, S. (Ed.) (2010). The ethical values of journalists. Field research among media professionals in Catalonia. Barcelona: Generalitat de Catalunya.

Alsius, S., Rodríguez-Martínez, R., y Mauri-Ríos, M. (2018). New formats and old crisis. En T. Eberwein, S. Fengler, y M. Karmasin (Eds.), The European Handbook of Media Accountability (pp.243-250). Abingdon, Oxon: Routledge.

Bertrand, C. J. (2018). Media ethics and accountability systems. Nueva York: Routledge.

Bichler, K., Harro-Loit, H., Karmasin, M., Kraus, D., Lauk, E., Loit, U., Fengler, S., Y Schneider-Mombaur, L. (2012). Best practice guidebook: media accountability and transparency across Europe. Disponible en http://www.mediaact.eu/fileadmin/user_upload/Guidebook/guidebook.pdf Brickman-Bhutta, C. (2012). Not by the book: Facebook as a sampling frame. Sociological Methods \& Research, 41(1), 57-88.

Bryman, A. (2016). Social research methods ( $5^{\mathrm{a}}$ ed.). Oxford: Oxford University Press.

Chaparro-Domínguez, M.A., Suárez-Villegas, J.C., y Rodríguez-Martínez, R. (2019). Media accountability and journalists: To whom do Spanish professionals feel responsible? Journalism Practice. https://doi.org/10.1080/17512786.2019.1655470 
Christians, C. G., Glasser, T. L., Nordenstreng, K., y White, R.A. (2009). Normative theories of the media. Journalism in democratic societies. Urbana: University of Illinois Press.

Craft, S., Vos, T., y Wolfgang, J. D. (2015). Reader comments as press criticism: Implications for the journalistic field. Journalism, 17(6), 677-693.

https://doi.org/10.1177/1464884915579332

Diaz Noci, J., Domingo, D., Masip, P., Micó, J. L., y Ruiz, C. (2012). Comments in news, democracy booster or journalistic nightmare: Assessing the quality and dynamics of citizen debates in Catalan online newspapers. International Symposium on Online Journalism, 2(1), 46-64.

Díez-Garrido, M., Campos-Domínguez, E., y Calvo, D. (2019). La transparencia de los partidos politicos como estrategia electoral. Una evaluación de sus promesas y sus páginas web. Trípodos, 44, 83-104. Disponible en http://www.tripodos.com/index.php/Facultat_Comunicacio Blanquerna/article/view/ $\underline{620}$

Fengler, S., Eberwein, T., Mazzoleni, G., Porlezza, C., y Russ-Mohl, S. (2014). Journalists and media accountability. An international study of news people in the digital age. Nueva York: Peter Lang.

Ferruci, P. (2019). The End of Ombudsmen? 21st-Century Journalism and Reader Representatives. Journalism \& Mass Communication Quarterly, 96(1), 288-307. https://doi.org/10.1177/1077699018805986

García-de-Torres, E., y Farmer, Y. (2017). Ética en los medios de comunicación: retos y oportunidades para la investigación. El Profesional de la Información, 26(2), 153-157. https://doi.org/10.3145/epi.2017.mar.01

Hallin, D.C., y Mancini, P. (2004). Comparing Media Systems: Three Models of Media and Politics. Nueva York: Cambridge University Press. 
Hanitzsch, T., Van Dalen, A., y Steindl, N. (2018). Caught in the Nexus: A Comparative and Longitudinal Analysis of Public Trust in the Press. The International Journal of Press/Politics, 23(1), 3-23. https://doi.org/10.1177/1940161217740695

Karlsson, M., Clerwall, C., y Nord, L. (2017). Do not stand corrected. Transparency and users' attitudes to inaccurate news and corrections in online journalism. Journalism \& Mass Communication Quarterly, 94(1), 148-167.

https://doi.org/10.1177\%2F1077699016654680

Luengo, M., Maciá-Barber, C., y Requejo-Alemán, J.L. (2017). Evaluating organisational ethics in Spanish news media. Journalism, 18(9), 1142-1162.

https://doi.org/10.1177\%2F1464884916643682

Maciá-Barber, C. (2006). La figura del defensor del lector, del oyente y del telespectador. Madrid: Universitas.

Masip, P., Ruiz-Caballero, C., y Suau, J. (2019). Active audiences and social discussion on the digital public sphere. Review article. El Profesional de la Información, 28(2), e280204. https://doi.org//10.3145/epi.2019.mar.04

Mauri-Ríos, M., y Ramon, X. (2015). Nuevos sistemas de rendición de cuentas de la información periodística. Una exploración del escenario online español. El Profesional de la Información, 24(4), 380-389. https://doi.org/10.3145/epi.2015.jul.04

McQuail, D. (1997). Accountability of media to society. Principles and means. European Journal of Communication, 12(4), 511-529.

https://doi.org/10.1177/0267323197012004004

Ramon-Vegas, X., Billings, A. C., y Rojas-Torrijos, J. L. (2019). Interviews with Former ESPN Ombudsmen / Public Editors Kelly McBride, Robert Lipsyte, and Jim Brady. International Journal of Sport Communication, 12(1), 28-35. https://doi.org/10.1123/ijsc.2018-0127

Ramon-Vegas, X., Mauri-Ríos, M., y Alcalá-Anguiano, F. (2016). Transparencia informativa, autorregulación y participación del público: Mural.com, Rue89.com y 
TexasTribune.org. Comunicación y Sociedad, 25, 101-125.

https://doi.org/10.32870/cys.v0i25.4423

Real Rodríguez, E. (2018). La profesión periodística ante sus retos éticos:

autorregulación profesional y comunicativa frente a regulación. La situación en

España. Estudios sobre el Mensaje Periodístico, 24(1), 341-360.

https://doi.org/10.5209/ESMP.59954

Rodríguez-Martínez, R., López-Meri, A., Merino-Arribas, A., y Mauri-Ríos, M. (2017). Instrumentos de rendición de cuentas en España. Análisis comparativo en Cataluña, Galicia, Madrid y Valencia. El Profesional de la Información, 26(2), 255-266.

https://doi.org/10.3145/epi.2017.mar.12

Rosique Cedillo, G., y Barranquero-Carretero, A. (2016). Analysis of media observatories in Spain. A tool for civil society in media reform processes. Revista Latina de Comunicación Social, 71, 443-469.

Suárez-Villegas, J.C., Rodríguez-Martínez, R., Mauri-Ríos, M., y López-Meri, A. (2017). Accountability y culturas periodísticas en España. Impacto y propuesta de buenas prácticas en los medios de comunicación españoles (MediaACES). Revista Latina de Comunicación Social, 72, 321-330.

Van der Wurff, R., y Schönbach, K. (2014). Audience Expectations of Media Accountability in the Netherlands. Journalism Studies, 15(2), 121-137. https://doi.org/10.1080/1461670X.2013.801679

Waisbord, S. (2019). The vulnerabilities of journalism. Journalism, 20(1), 210-213. https://doi.org/10.1177\%2F1464884918809283

Zelizer, B. (2018). Epilogue: Timing the study of news temporality. Journalism, 19(1), 111-121. https://doi.org/10.1177\%2F1464884916688964 